\title{
Research into induced seismicity in the Groningen field - further studies
}

\section{Jan-Dirk Jansen ${ }^{1, *}$ \& Rien Herber ${ }^{2}$}

1 Department of Geoscience and Engineering, Delft University of Technology, Stevinweg 1, $2628 \mathrm{CN}$ Delft, the Netherlands

2 Energy and Sustainability Research Institute, University of Groningen, Groningen, the Netherlands

* Corresponding author. Email: j.d.jansen@tudelft.nl

Manuscript received: 16 May 2017, accepted: 27 July 2017

\section{Abstract}

Further research into seismicity caused by natural gas production from the Groningen field is necessary to improve the assessment of seismic risk and develop means to control and reduce it. Research into subsurface aspects is primarily of relevance to assess the seismic hazard component in the cause-and-effect chain that governs the seismic risk. It requires a wide range of research activities that can be broadly classified as follows:

- Increasing understanding of the physical mechanisms that govern production-induced seismicity, in particular source mechanisms, compaction behaviour, propagation of energy to the surface, and the effects of fluctuating production.

- Reducing uncertainty by acquiring additional field data to improve statistical inference, and developing statistical methods and procedures that can cope with the non-stationary nature of the process.

- Developing tools and techniques to improve risk management, and support operational control and policy measures under uncertainty.

An essential requirement for further research will be the possibility of developing competing theories for many aspects of the modelling chain. This requires an overall hazard and risk assessment methodology that can accommodate multiple models, and an organisational structure that facilitates the comparison of competing approaches while safeguarding their independent development. This will have to be supported by the availability of reliable data via shared databases. Finally, the scientific community should be prepared to make a major effort to translate their research results into popular scientific versions in order to keep stakeholders abreast of progressive insight into the origin, predictability and prevention of induced seismicity.

Keywords: gas production, Groningen, induced seismicity, mechanistic models, nonstationary statistics, statistical models

\section{Introduction}

Research into seismicity induced by natural gas production from the Groningen field has recently experienced very strong growth, driven by increasing seismic activity in the field, the associated safety risks and large societal impact. For many years, however, research into seismicity was considered less relevant than research into another production-induced geomechanical phenomenon: subsidence. In the Netherlands, a country situated largely below sea level, water management is of prime importance, and the prediction of subsidence resulting from oiland gas extraction has therefore always received attention: any amount of subsidence in an area requires a proportionate increase in the height of the dikes to prevent that area from flooding. Early subsidence studies for the Groningen field were published in the 1960s, and updated studies, accompanied by comprehensive monitoring activity, have since continued with a frequency of every five years.

Since 1986, relatively small earthquakes were observed above producing gas fields in the north of the Netherlands, with the first registered event above the Groningen field occurring in 1991. For an overview of the resulting research activities, which were of limited extent, and an account of the controversy about the cause of the earthquakes, we refer to a report of the Dutch Safety Board (0VV, 2015). A Dutch Government-sponsored study, published in 1993, concluded that the earthquakes were indeed of non-tectonic origin and resulted from gas production. However, the general feeling in those days was that their magnitude would never reach a level that would generate serious concern. Nevertheless, it was decided to install a dedicated monitoring 
network of accelerometers, managed by the Dutch national meteorological institute KNMI, capable of registering events with a magnitude above 1.5. Only after the Huizinge event of magnitude 3.6, in 2012, did a sense of urgency develop, resulting in a large series of studies. Many of these were performed by the Nederlandse Aardolie Maatschappij (NAM), the operator of the field, or commissioned by NAM to third parties, as documented in the Groningen Winningsplan (NAM, 2016) and summarised in another paper in this Special Issue (Van Elk 2017). Numerous studies were also performed by other parties, in particular TNO and KNMI, whilst it was also decided to significantly expand the KNMI monitoring network.

However, an integrated large-scale research effort into all aspects of the observed production-induced seismicity in the Groningen field, independently of NAM, has not yet been started. This is notwithstanding the fact that already in 2012 a joint effort of Dutch universities and applied research institutes resulted in a proposal to the Dutch Government for such a National Induced Seismicity Platform, which unfortunately did not lead to any tangible results. In 2015, the Dutch Safety Board issued a report in which it recommended installing 'a structural and long-term research programme within which integral and independent scientific and applied research into these problems is performed' (see OVV, 2015). This conclusion was subsequently adopted by the Dutch Parliament, and the initiation of such a programme, 'Kennisprogramma Effecten Mijnbouw (KEM)' (Knowledge Program Effects of Mining), was agreed to by the Minister of Economic Affairs in 2016. It is expected that in the course of 2017 the programme's first activities will be performed, with a strong focus on questions that can be answered on shortterm as well as medium-term timescales (months to a couple of years). Contributions are therefore primarily expected from applied research institutes, and to a lesser extent from universities, both in the Netherlands and abroad. More or less independent of this development, the Netherlands Organisation for Scientific Research (NW0) has been preparing a funding scheme for more long-term focused, fundamental research, primarily aimed at Dutch universities. This development, DeepNL, is expected to kick off in 2018.

\section{Research needs}

\section{Scope of this overview}

Current research into the subsurface aspects of productioninduced seismicity in Groningen is primarily aimed at quantifying the seismic hazard, typically expressed in terms of the probability of exceeding a certain peak ground acceleration (PGA) resulting from seismic events. In combination with the dynamic response of buildings and other surface objects to these accelerations, it is then possible to quantify the seismic risk, expressed in terms of statistics of fatalities, injuries or damage. Although the division between subsurface and surface contributions to the seismic risk is sometimes suboptimal, we will maintain that division and only address the need for further studies related to the subsurface aspects of induced seismicity in Groningen in line with the scope of the Netherlands Journal of Geosciences. An overview of the associated research topics that we identified is given in Table 1, together with a classification of laboratory experiments, field experiments and theoretical research (interpretation, numerical modelling and theory development).

\section{Overarching themes}

Limited basis for statistics The only comprehensive, presently available model to quantify seismicity-related risk in Groningen is the one developed by NAM (Van Elk 2017). Several key components, such as the seismic source model and the ground motion prediction model, strongly rely on statistics. Unfortunately the number of recorded seismic events in Groningen is limited because of the relatively short period that seismicity has been observed and the limited resolution of recorded events in the early stages of the monitoring programme. This introduces significant uncertainties in the prediction model.

Stationarity versus transient behaviour Another complicating problem is that a large part of geophysical theory is based on the assumption that seismicity is stationary, i.e. events may occur randomly but their statistics stay constant over time. This is obviously not the case for Groningen, where seismicity has started only very recently and will end again very soon (on geological timescales), some time after the end of the gas production period. A complicating factor results from the various changes that have been made in the operation of the Groningen field. In particular the reduction of gas production in recent years and the change from production with strong seasonal fluctuations to production with a much flatter profile make predictions based on stationary statistics even more uncertain. The induced seismicity process in Groningen is therefore transient and statistics based on the assumption of stationarity do not necessarily apply.

Statistics versus first-principles modelling A theoretical alternative is the use of first-principles modelling, also referred to as mechanistic modelling, in which predictions are (to a large extent) based on numerical models containing codified physical laws and results from controlled laboratory or field experiments. Unfortunately, attempts to use such first-principles modelling to predict seismicity in Groningen have not been successful to date. An approach to develop predictive models, preferably with as much physics and as little statistics as possible, is therefore necessary. However, a key component of any research programme into induced seismicity in Groningen will have to deeply investigate physical mechanisms with the aim of obtaining quantitative predictive models where possible. 


\begin{tabular}{|c|c|c|c|}
\hline \multirow[b]{2}{*}{ Research topic } & \multicolumn{3}{|c|}{ Research type } \\
\hline & Lab & Field & Theory \\
\hline \multicolumn{4}{|l|}{ Understanding the source } \\
\hline Monitoring of seismic events & & $\mathrm{X}$ & \\
\hline Pinpointing the location of seismic events & & $\mathrm{X}$ & $\mathrm{X}$ \\
\hline Determining in situ stresses below the reservoir & & $\mathrm{X}$ & $\mathrm{X}$ \\
\hline Understanding friction behaviour of reservoir and non-reservoir rock in faults & $\mathrm{X}$ & & $\mathrm{X}$ \\
\hline Understanding (time-dependent) deformation mechanisms of reservoir rock & $\mathrm{X}$ & $\mathrm{X}$ & $\mathrm{X}$ \\
\hline Understanding (time-dependent) mechanical properties of salt and anhydrite & $\mathrm{X}$ & $\mathrm{X}$ & $\mathrm{X}$ \\
\hline Understanding the direct and indirect effects of pore pressure changes on fault stresses & $\mathrm{X}$ & & $\mathrm{X}$ \\
\hline Quantifying the effects of pressure propagation through faults outside the reservoir & & & $\mathrm{X}$ \\
\hline Assessing the hazard related to cyclic (seasonal) production and sudden rate changes & & & $\mathrm{X}$ \\
\hline Understanding the (dynamic) areal propagation of stress release along fault planes & & $\mathrm{X}$ & $\mathrm{X}$ \\
\hline Quantifying the effect of multiple-event and aftershock generation by wave propagation & & $\mathrm{X}$ & $\mathrm{X}$ \\
\hline \multicolumn{4}{|l|}{ Quantifying propagation to surface } \\
\hline Gathering further earthquake statistics & & $\mathrm{X}$ & \\
\hline Gathering further data on near-surface soil conditions & $\mathrm{X}$ & $\mathrm{X}$ & \\
\hline Testing the transient and long-term time-dependent behaviour of soils & $\mathrm{X}$ & & \\
\hline Numerically modelling wave propagation to surface & & & $\mathrm{X}$ \\
\hline Data-driven prediction of wave propagation to surface & & $\mathrm{X}$ & $\mathrm{X}$ \\
\hline Assessing soil properties and soil-foundation interactions & $\mathrm{X}$ & $\mathrm{X}$ & $\mathrm{X}$ \\
\hline Further developing Groningen-specific GMPEs & & & $\mathrm{X}$ \\
\hline \multicolumn{4}{|l|}{ Reducing uncertainty } \\
\hline Assessing the applicability of existing seismological theory to transient seismicity & & & $\mathrm{X}$ \\
\hline Improving statistical models with mechanistic simulation & & & $\mathrm{X}$ \\
\hline Improving uncertainty quantification with competing models & & & $\mathrm{X}$ \\
\hline Developing a risk assessment methodology that can accommodate multiple models & & & $\mathrm{X}$ \\
\hline \multicolumn{4}{|l|}{ Managing the reservoir } \\
\hline Understanding control mechanisms & $\mathrm{X}$ & & $\mathrm{X}$ \\
\hline Developing statistical methods & & & $\mathrm{X}$ \\
\hline Developing methods for pressure control & $\mathrm{X}$ & & $\mathrm{X}$ \\
\hline Developing protocols & & & $\mathrm{X}$ \\
\hline
\end{tabular}

Competing theories An essential aspect will be the need to develop competing theories for many aspects of the modelling chain and resist the temptation to harmonise developments or prune the tree of possible options too early. Such a parallel approach is in conflict with the need to obtain rapid predictions and support operational or policy decisions on short timescales. It is, however, essential to develop fundamental understanding of the mechanisms involved and to avoid remaining on a wrong track simply because alternatives have been lost from view at an early stage.

In addition to the need to diversify theory development, the limits of operational scenarios should be chosen as wide as scientifically relevant, and not be limited to what is economically desirable. This is another aspect where there is a clear discrepancy between scientific research objectives on the one side, and economic and policy aspects on the other.
Open access to data The call for a 'totally' independent research programme into gas-production induced earthquakes in Groningen is understandable, but in practice probably impossible. A very large proportion of the present body of scientific knowledge has been developed by operator staff, in particular from NAM and Shell, or others commissioned by NAM. Additional knowledge has been developed by research institutes, but essential information for these models has often been obtained from NAM. Unfortunately, just making operator models available to independent researchers is usually not meaningful, because the programmes are typically incomprehensible without expert advice from the parties who generated them.

When it applies to data, the situation is different. During recent years a significant monitoring and data acquisition programme has been set up for the Groningen field. It is essential that these data are readily accessible in systematic databases in 
a shared format. Examples are recorded soil responses, downhole seismicity signals at reservoir levels, strain state from fibre optics in wells, core data, cone penetration test data, etc. The databases also need to contain reliable metadata such as coordinates, depths, time, date, etc. This will make it possible to compare research results on an appropriate basis: discussion should be on interpretation of results, not on data.

Accessibility and acceptability of research results We are convinced that the complex reality of the induced seismicity in Groningen can only be unravelled when research results are shared in a timely manner. This means that models, experiments and interpretations from various researchers can be compared in order to deepen scientific understanding and promote progressive insight. Arguments for confidentiality or time delays due to publication in scientific magazines will hinder such progress, which is not acceptable in view of the urgent need for society to come up with solutions for predicting/preventing tremors. Therefore, the scientific programmes mentioned above, such as DeepNL and KEM, should provide peer review protocols, since these are proven tools for achieving scientific integrity and quality.

Outreach to the general public forms a very important element in disseminating the improved insights. The scientific community needs to make a major effort to translate its research results into popular scientific versions in order to keep stakeholders abreast of progressive insights into the origin, predictability and prevention of induced seismicity. This is a societal responsibility which should be taken seriously by scientific investigators.

\section{Understanding the source}

Induced and triggered events Within the scientific community there is general agreement that there are several possible chains of causality between gas production and seismic events. In all of them, gas production from a porous reservoir leads to a decrease in reservoir pore pressure and therefore an increase in 'effective' stresses in the reservoir rock. The increase in vertical effective stress causes compaction of the reservoir rock because of temporary (elastic) and permanent (plastic or brittle) deformations in between and inside the grains. Compaction in the reservoir will lead to displacements inside the reservoir and likely also in the layers directly above and below, which may lead to subsidence as observed at the surface. Moreover, the combined effect of changes in pore pressure and the resulting compaction may trigger seismic events (i.e. sudden releases of energy) in faults that are near-critically stressed because of tectonic movements unrelated to gas production. In addition to triggering earthquakes, pore pressure reduction may also induce them, by being the very cause of the build-up of shear stresses and/or the reduction of normal stresses on faults, either directly or in combination with the effects of compaction. This is possible, for example, in the case of unequal compaction at two sides of a sealing fault, or in the case of a fault with a significant throw such that compacting reservoir rock is in direct contact with stationary nonreservoir rock. However, since fractures may also be pathways for the transmission of pressures, more complex scenarios are possible, in which pressure depletion and compaction lead to induced earthquakes in faults outside the reservoir. Which source mechanisms are at play in Groningen and where exactly the events are located is still a matter of scientific debate.

Pinpointing event locations An important related research question is therefore 'Where do the seismic events occur?' Answering this question requires continued geophysical field measurements, primarily from the existing monitoring network, especially at reservoir level, as well as improved interpretation. This involves not only computationally intensive numerical techniques for full waveform analysis, but also intensive cooperation with geomechanics experts, reservoir engineers and geologists to understand the interaction between wave propagation, stress field and geology. A potentially important role may be played by emerging geophysical methods, in particular interferometry, to obtain additional information from noisy signals. Related research aspects concern the in situ stress state, especially at greater depths below the reservoir. Although it is generally accepted that the Groningen field is located in a tectonically inactive area, the presence of critically stressed faults at greater depth is still a hypothesis that has not been conclusively rejected.

Understanding geomechanics A key uncertain aspect in the generation of seismic events, requiring more research for Groningenspecific conditions, is the friction behaviour of rock surfaces in faults, both inside and outside the reservoir, and its dependence on stress state and deformation rate. This is directly related to the nucleation behaviour of earthquakes (i.e. the development in time and space of multiple events). This is an active research topic in the geophysical community which has primarily been developed for steady-state tectonic situations and hence requires revision for transient production-induced conditions as present in Groningen.

Another key geomechanical uncertainty concerns the deformation behaviour of the reservoir sandstone, including compaction in response to pore pressure reduction. The nonlinear and non-reversible mechanisms of matrix deformation and their dependence on time are only partly understood. A related uncertainty concerns the petrophysical parameters of rock outside the reservoir, in particular those related to the plastic behaviour of the salt and the stiffness of the anhydrite layers just above it.

Apart from research into constitutive relationships that govern friction and deformation, there is also a need for a better understanding of the geomechanical response (i.e. the development of stresses, strains and deformations) in the reservoir and its surroundings as a function of initial and boundary conditions and production-induced pore pressure changes, especially in and around faults. Improved insight into all these 
geomechanical aspects is essential to answer the question 'Do reduced production rates lead to a reduced seismic activity over the producing life of the reservoir or only to a delay in the occurrence of seismic events?'

Quantifying the effects of dynamics The induction and triggering mechanisms described above may occur quasi-statically, i.e. such that the dynamic response of the reservoir to pressure depletion before the occurrence of a seismic event is much slower than the typical dynamics of a reservoir responding to changes in production. The latter is governed, at least to first order, by the slow poro-elastic behaviour of the reservoir, i.e. by the dynamics of fluid pressures in the reservoir rock and the faults. Particularly relevant to the Groningen reservoir is the question 'Can fluctuations in production, either resulting from the sudden opening or closing of wells, or from seasonal fluctuations in gas production, trigger seismic events?' After occurrence of an event, the dynamic response of the reservoir is governed by the fast poro-elastic behaviour of the reservoir, in which the dynamics of the moving rock, in combination with the pressure dynamics, play a leading role. Stress waves travelling through the reservoir may trigger new events at other locations, a phenomenon well known from tectonic earthquakes. Such events may occur in the same fault where the initial event took place, leading to a gradually extending region of partial stress release in that fault, or in faults at a larger distance. The mechanics of fault unloading and the statistics of multiple (near-immediate) events or (time-delayed) aftershocks for production-induced seismicity in Groningen are both areas where further research is required.

\section{Quantifying propagation to surface}

Developing ground motion prediction equations 0ne of the major contributions to uncertainty in the seismic hazard for Groningen, and thus to uncertainty in the seismic risk, is related to the propagation of seismic energy to surface (Van Elk 2017). Within the geophysical community the development of ground motion prediction equations (GMPEs) is a well-developed subject and much effort has already been put into the development of a Groningen-specific GMPE. In particular, the soil conditions in the top few hundred metres in Groningen have a large effect on the accelerations observed at the surface because the stiffness and damping properties determine to what extent amplitudes and frequency content of the travelling waves are modified. In a major geological modelling campaign, Deltares has made an inventory of soil conditions in the top soil layers in the area affected by earthquakes in Groningen. Although this has reduced the uncertainty in the Groningen GMPE, there is significant scope to further improve its predictive capacity, e.g. through data-driven methods. Moreover, current GMPEs focus on shear wave motions, since the horizontal component affects the movement of and damage to buildings the most. However, the vertical component also needs further attention, as observed in several damage cases in the Groningen area.

Complementing empiricism with first-principles models Traditional GMPE development has a strongly empirical character and employs statistics, which, unfortunately, for Groningen are only available to a limited extent because of the relatively short seismic history of the field and the limited resolution, in terms of magnitude, of early seismological measurements. Use of statistics from other areas is also only possible to a limited extent because of Groningen-specific conditions (relatively shallow earthquakes, thick overlying salt strata, soft soil conditions at the surface, etc.). In addition to further data gathering, both on earthquake statistics and soil properties, there seems to be ample scope for complementary first-principles-based studies. These will involve large-scale numerical simulation of wave propagation in combination with advanced uncertainty quantification methods.

Assessing soil properties and soil-foundation interactions 0ther related efforts include laboratory testing of soil response, and the assessment of soil-foundation interaction (piled foundations, deep excavations, subsurface parking structures, cellars). This is a research topic where just expressing the seismic hazard in terms of ground accelerations at the surface is not very meaningful, and where a strict separation between 'surface' and 'subsurface' aspects of induced seismicity is not possible.

\section{Reducing uncertainty}

Improving statistical models with mechanistic simulation Many aspects of the cause-and-effect chain of induced seismicity depend on statistical observations. Earlier, we discussed the inherent limitation of statistical models for prediction of seismicity and the related hazard in Groningen, because of the limited historical data and the essentially transient nature (on geological timescales) of the underlying geophysical processes. Nevertheless, statistical models will remain an essential part of any comprehensive research programme into induced seismicity because many aspects are beyond the capabilities of current mechanistic models. Moreover, a vast body of earthquake science exists that relates the frequency and magnitudes of events to time series of observations and implicitly or explicitly includes underlying physical mechanisms. Research into statistical models should therefore focus on assessing the applicability of existing methods, often developed for deep, naturally occurring earthquakes of a stationary nature, to the relatively shallow, production-induced, transient seismicity in Groningen. The use of large-scale numerical simulation of phenomenological or detailed physics may in itself never result in predictive models, but may be used to develop insight into the range of validity of the current statistical models, and possibly to extend that range beyond its current uncertain limits. 
Improving uncertainty quantification with competing models As mentioned above, an essential aspect of further research should be the development of a variety of competing mechanistic, statistical or hybrid models. This may involve models for, e.g., the in situ stress state, time-dependent compaction behaviour, dynamic behaviour of materials, fracture and slip propagation in faults, fluid flow and pressure diffusion across faults, event occurrence related to geomechanics, energy propagation to surface, and other elements in the cause-and-effect chain. Apart from using these competing models, and their underlying scientific hypotheses, to increase understanding, they may also be used to improve uncertainty quantification. This requires an overall hazard and risk assessment methodology that can accommodate multiple models, both conceptually and mathematically, and an organisational structure that facilitates the comparison of competing approaches whilst safeguarding their independent development.

\section{Managing the reservoir}

Developing control procedures Several of the measures to reduce the risk of induced seismicity from gas production in Groningen involve changes in operation of the field. This concerns restrictions on production rates from individual wells or clusters, spatial optimisation of offtake over the field, reduction of production variations in time, or measurement-and-control protocols to modify production in response to observable parameters such as seismicity rate or event magnitude. Some of these measures were initiated by the operator, while others were imposed by Dutch Governmental authorities. However, the underlying rationale for many of these measures is often based on assumptions or hypotheses rather than proven theory.

An important part of further research should therefore be the development of a body of theoretical insight and experimental evidence to justify and optimise production control measures. This research topic is strongly linked to better understanding the source mechanisms, as described above, especially understanding the (time-dependent) geomechanical behaviour of the reservoir, and quantifying the effect of dynamics. It should also involve research into control-theoretical aspects, such as controllability and observability of reservoir state variables (pressures, temperatures, displacements, velocities), identifiability of parameters under uncertainty, and the development of stochastic optimal control procedures.

Developing statistical methods Because of inherent uncertainties in the earthquake nucleation process, decision criteria will require a statistical basis. Also the justification of control measures in response to observed events (protocols) requires a statistically sound approach to determine triggering levels and response measures. Statistics also plays an important role in the assessment of the effect of control measures, both in time and in space. Moreover, statistics could be used to design controlled experimental operational procedures, and give a steer so that frequent changes in control policy do not make it impossible to evaluate their effect in a statistically significant manner. Because of the transient nature of the induced seismicity process, it may be necessary to develop dedicated statistical techniques for these applications.

Controlling pressures Reinjection of fluids into the reservoir, to entirely or partially replace the produced gas, is an apparently obvious solutions to eliminate the root cause of induced seismicity. Research efforts to date indicate that the potential disadvantages outweigh the benefits; identified problems include increased risk of triggering seismicity because of locally increased pressures around injection wells and pore pressure propagation or lubrication effects in faults. Growing insight into the source mechanisms and reservoir dynamics may offer opportunities to revise these findings.

\section{Conclusion}

A broad range of further research, preferably based on competing hypotheses, is necessary to improve the assessment of seismic risk in Groningen and develop means to control and reduce it. Key elements are an increased understanding of physical mechanisms in combination with improved statistical methods to cope with processes of an essentially non-stationary nature.

\section{References}

NAM, 2016. Study and data acquisition plan induced seismicity in Groningen - update post Winningsplan 2016, Parts 1 and 2. Report EP201604200072. Nederlandse Aardolie Maatschappij (Assen). Available at www.nam.nl/feitenen-cijfers/onderzoeksrapporten.html.

oVV, 2015. Aardbevingsrisico's in Groningen - onderzoek naar de rol van veiligheid van burgers in de besluitvorming over de gaswinning (1959-2014). Onderzoeksraad voor Veiligheid (The Hague). Available at www.onderzoeksraad.nl.

Van Elk, J. 2017. Hazard and risk assessments for induced seismicity in Groningen. Netherlands Journal of Geosciences / Geologie en Mijnbouw, this issue. 